\title{
An RDF NetAPI
}

\author{
Andy Seaborne \\ Hewlett-Packard Laboratories, Bristol \\ andy_seaborne@hp.com
}

\begin{abstract}
This paper describes some initial work on a NetAPI for accessing and updating RDF data over the web. The NetAPI includes actions for conditional extraction or update of RDF data, actions for model upload and download and also the ability to enquire about the capabilities of a hosting server. An initial experimental system is described which partially implements these ideas within the Jena toolkit.
\end{abstract}

\section{The Need for a NetAPI for RDF}

Part of fulfilling the vision of the Semantic Web is the exchange of RDF[1] data between computer systems. The web enables the reuse of resources so that people, and now systems, can obtain, combine and process information from other systems without explicit producer-consumer relationships being set up.

One precursor for the Semantic Web to achieve critical mass will be a common framework for accessing RDF data, one sufficiently common that the majority of applications will use it, the majority of publication host systems support it and only specialized applications will choose to develop their own protocols.

Given a common protocol, the network effect of bringing in more and more semantic applications can start. Application developers can concentrate on the application, and not on the plumbing. Tool builders (server and client side) do not need to support multiple protocols, and can target client-side or server-side environments. Publishers can know that many systems and applications can access their RDF information.

In this paper, we argue for the use of "conditional GET" and "partial PUT" as the central operations for accessing RDF datasources. In the outline framework, we incorporate existing actions of HTTP [2], such as HTTP GET and PUT, as the simplest level but, as the semantic web grows, and as large metadata repositories appear, the paradigm shifts to one that is more like database access than web page download.

We have built a small experimental system that demonstrates part of the NetAPI. We do not propose specific protocols here and this is not the only attempt to create remote interaction with RDF data. We are investigating what it would take to create mass deployment on the current web infrastructure. 


\section{Design Challenges}

One challenge is to balance simplicity of implementation, leading to widespread deployment, with adequate functionality. We want a simple, widely used infrastructure.

In any complex system, there is often a gap between the requirements of the application and the capabilities of the general infrastructure that is properly met by domain-specific sub-systems. These domain specific systems appear as "applications" to the infrastructure and as "infrastructure" to the end applications.

Another challenge in the design of a NetAPI for RDF-based systems is to provide a useful protocol that separates the evolution of client and server software in a community that is pulled from diverse research and industrial domains so the protocol should be simple and domain independent.

We envisage further protocols, both on top of the common access framework proposed here and also more specialized protocols for specific uses where the common access framework is insufficient. A basis for a general system will rarely meet the needs of all possible domains directly.

We also want to utilize as much of the common web infrastructure for the access of RDF data, using existing protocols and existing server systems. This decreases the barriers to deployment.

\section{Outline Framework}

We limit the discussion here to operations on single models, viewed as collections of RDF statements. Applications will also want to merge data sources, issue queries across several data sources or (consistently) update multiple sources. This should be considered for possible later addition: the focus here is to identify a simple set of clearly defined operations, which will boost the adoption of the semantic web.

There are 3 categories of operations, which have been identified:

- Operations on the RDF data itself (query, update)

- Transfer of complete sets of RDF data as RDF models.

- Operations relating to the hosting server, such as enquiring about its capabilities and the range of operations on particular RDF stores.

We do not see the basic operations of RDF data as a fetch but one that is conditioned to select a subset of the data available. For this we need selection languages - we do not anticipate that one language will meet a sufficiently wide set of needs at the moment so we make the selection language a parameter of the operation. In future, we hope that a single language emerges with sufficient coverage that both RDF data providers and RDF data consumers can expect to find support for it in all toolkits.

\section{Server Capabilities}

The execution model is one where there are client-initiated actions. Some of the operations have optional parameters so client-side toolkits and applications may need to enquire about the capabilities offered by a host server and about the legal operations and parameters on each of the data sources hosted at that server. This 
reduces to the server needing to provide information about its capabilities, both overall and with regard to specific RDF data sources.

\section{Model Operations}

Operations that fetch or store whole RDF models are the simplest level of operation for an RDF data source and these naturally map to the HTTP operations GET and PUT. These are already supported by existing web servers, where the data source is an RDF document. Simple extension to retrieving the metadata about a web object, instead of the object itself, based on, say, the MIME type is also natural.

Abstractly, the operations are:

- GET (model, format)

- PUT (model, format)

These operations give a sea of RDF models with client systems loading RDF data and performing the extraction and merging of data at the client. These may or may not map directly to the HTTP verbs PUT and GET.

Such whole model operations are not suitable where the quantity of RDF information is large, yet the actual information required by the client is small (for example: data about a publication from a digital library). Nor does it provide for the update of data source, only complete replacement.

For these operations, we need operations acting on client-defined sets of RDF statements, not the whole model (which is a set defined by the server).

\section{Triple operations}

In order to operate on specific data sources we propose operations:

- GET-TRIPLES (model, language, condition, format)

- UPDATE-TRIPLES (model, data-to-delete, data-to-add)

These are not proposed as new verbs for HTTP.

The language parameter means that we can have a variety of languages for extraction of data from RDF sources: we anticipate that this will usually be a query language and there are advantages in providing a common mechanism where possible. However, we recognize that a single language cannot meet the needs of, say, query and of inferencing rules systems at this stage.

The choice of a "delete-add" operation allows for consistent bulk update of a data source. The deletions are performed before the additions.

The "format" parameter allows variable in the output format even for the same query language (examples: returning bound variables or the RDF subgraph that matched the query: providing XML or N-triple for update).

\section{An Experiment: An RDF Server}

The experimental system implements the operations of GET-TRIPLES and UPDATETRIPLES. 


\section{Protocol Style}

We seek a simple, fixed, set of operations that can be widely implemented, enabling client and server toolkits to evolve independently (see [8] for a discussion of protocol styles).

At the same time, we wish to reuse the existing deployed web infrastructure. To maximize this, we currently build on top of HTTP POST, partly in the style of SOAP [9], but, unlike SOAP, we restrict the operations strictly to those outlined in this paper. The protocol uses RDF model transfer as the message format; we do not suggest that this represents the best choice for a production protocol - it is an outbreak of "next-bench" syndrome.

\section{Protocols and Query Language}

The protocol consists of a number of layers (from lowest to highest in the stack):

1. A model exchange layer

2. A request-response layer

3. A query layer for conditional GET

Each operation is encoded as a single RDF model to give SOAP-like operation, with higher levels on the stack attaching their protocol information via properties to the message URI. Both body and header information are contained in the same RDF model. The request-response layer simply matches responses to the request that caused them.

\section{Conditional Selection Language}

The conditional selection language is RDQL, an implementation of SquishQL[3] for the Jena toolkit[7]. SquishQL is an SQL-like query language that matches a graph pattern to a data source; filter functions can restrict the values of variables. RDQL returns both bound variables and the triples that caused the binding. SquishQL has been variously implemented [4 5.6].

The query request contains an RDQL query, complete with URI to specify the data source. The response contains the variable bindings in an RDF data structure, and the triples that cause the binding are associated with the particular binding using reification. The return format is N-Triple because there are usually shared bNodes.

\section{Client Interface}

The client-side implements remote query operations and remote update for the Jena toolkit. The same Java interface is provided for remote query as for query of local models by implementing the same interface. The URI of the data source in included in the query itself as usual. The client creates a remote query engine that takes the URL as the location of the remote server.

The client processes the results through an iterator in the same programming paradigm as for local queries. The only difference is the creation of the query execution object; a remote query engine takes a location URL.

\section{Server}

The server implementation is a conventional servlet in a servlet-container web server, with HTTP POST, and the reply to the POST, used as the transport for model exchange. Both GET-TRIPLES and UPDATE-TRIPLES are supported. 
The server is configured with a set of RDF models, each with its own URI (not tied to the host server), the location within the host of the data for the model and the URL of the servlet. This separation of the name of the data source (the model URI) from the location of the action (the URL where the operations are performed) allows systems administrators control over the location of models on the host systems and hides information such as filenames and JDBC connection URLs. RDF data sources can be relocated on the server without change to the client code.

\section{Review}

This experimental system is not finished. The use of the query and batch update paradigms for processing information is well suited to the network environment where operations are coarser grained and higher latency than API calls directly on a model implementation. The application writer sees the conventional query result processing paradigm that masks the network details and see explicit "batch and execute" for update.

One difference is that the client application can not fully mix the query calls with Jena API calls as is possible when the local model is available. This is because only a partial copy of the RDF data is available at the client as a model.

\section{Next Steps}

We plan to use the experimental RDF server in the construction of RDF driven applications, to test the protocol structure, the client programming paradigm and the server-side issues in managing RDF data.

\section{References}

1. Ora Lassila, Ralph R. Swick (editors), "Resource Description Framework (RDF) Model and Syntax Specification”, 22 February 1999.

2. RFC2616, "Hypertext Transfer Protocol -- HTTP/1.1"

3. L. Miller, A. Seaborne, A. Reggiori, "Three Implementations of SquishQL, a Simple RDF Query Language", submitted to ISWC2002.

4. A. Seaborne, RDQL - RDF Data Query Language. RDQL grammar: http://www.hpl.hp.com/semweb/rdql-grammar.html

5. L. Miller, "Inkling: RDF query using SquishQL", web page: http://swordfish.rdfweb.org/rdfquery/

6. A. Reggiori, D. W. van Gulik, RDFStore, http://rdfstore.sourceforge.net

7. The Jena toolkit: HPLabs Semantic Web activity, http://hpl.hp.com/semweb/

8. R.T. Fielding, "Architectural Styles and the Design of Network-based Software Architectures". Doctoral dissertation, University of California, Irvine, 2000.

9. M. Gudgin, M. Hadley, J. Moreau, H.F. Nielsen (editors), "SOAP Version 1.2" (working draft), http://www.w3.org/TR/2001/WD-soap12-part1-20011217/ 